\title{
Influencia de Dientes Remanentes en la Presencia y Morfometría de Forámenes y Canales en Relación a las Espinas Mentales
}

\author{
Influence of Remnant Teeth in the Presence and Morphometry \\ of Foramina and Canals in Relation to Mental Spine
}

Felipe Cáceres*; Valeria Ramírez ${ }^{* *}$ \& Reinaldo Soto*

CÁCERES, F.; RAMÍREZ V. \& SOTO, R. Influencia de dientes remanentes en la presencia y morfometría de forámenes y canales en relación a las espinas mentales. Int. J. Morphol., 32(1):106-111, 2014.

RESUMEN: La zona anterior de la mandíbula, con frecuencia es intervenida desde el punto de vista quirúrgico (instalación de implantes oseointegrados, mentoplastias, genioplastias, profundizaciones de vestíbulo y flanco lingual, fracturas, etc.). Se ha descrito la presencia de vasos que penetran la tabla ósea lingual en la zona mediana mandibular, los cuales se relacionan con forámenes vasculares. La pérdida dentaria en la zona anterior mandibular podría influir en el tamaño de los forámenes y canales debido a la degeneración de los vasos incisivos provenientes de la arteria alveolar inferior. El objetivo fue evaluar la relación entre presencia y ausencia de dientes remanentes en el grupo $\mathrm{V}$ con la presencia y morfometría de forámenes y canales ubicados en cercanía a las espinas mentales. Se estudiaron 95 mandíbulas humanas secas, con sexo y edad indeterminada. Se midió el área de los forámenes y la longitud de los canales utilizando el programa Photoshop CS3 extended. Se obtuvo un total de 53 mandíbulas dentadas en el grupo V y 42 mandíbulas desdentadas en el grupo V. El 95,7\% de la muestra tuvo al menos un foramen en relación a las espinas mentales. Se vio que los forámenes con mayor frecuencia de aparición fueron los superiores a las espinas mentales superiores en 75 mandíbulas, forámenes inferiores a las espinas mentales inferiores en 72 mandíbulas y los forámenes entre las espinas mentales en 26 mandíbulas. No se encontró asociación entre la presencia y ausencia de dientes con la frecuencia de forámenes en las tres posiciones estudiadas. Se observó que el foramen inferior a espinas mentales tuvo un diámetro mayor en mandíbulas dentadas (valor p= 0,0194) y la longitud del canal superior a las espinas mentales superiores fue significativamente más largo en mandíbulas desdentadas (valor p=0,0206).

PALABRAS CLAVE: Foramen lingual; Canal lingual; Espinas mentales.

\section{INTRODUCCIÓN}

La presencia de forámenes y canales en relación a las espinas mentales ha sido descrita en múltiples artículos, incluso siendo descrita en la población chilena (McDonnell et al. 1994; Flanagan, 2003; Liang et al., 2004; Liang et al., 2006; Soto et al., 2012). La importancia de estos elementos anatómicos radica en que el principal componente que viaja por ellos es de características arteriales (Lustig et al., 2003). Se ha afirmado que estos vasos pueden provenir ya sea de la arteria sublingual (rama de la arteria lingual) o de la arteria submentoniana (rama de la arteria facial) (Romanos et al., 2012). Esto explica la presencia de múltiples reportes de casos donde se ha visto hemorragias de importancia asociadas a actos quirúrgicos como instalación de implantes sinfisiarios, toma de injertos de mentón, genioplastias, colgajos linguales, etc., incluso comprometiendo la vida de los pacientes (Flanagan; Isaacson, 2004; Kalpidis \& Setayesh, 2004; Rosano et al., 2009). El destino de estos vasos finalmente se ha atribuido al tejido óseo mandibular de la zona, periodonto y pulpa de los dientes anteroinferiores, actuando como irrigación complementaria a la entregada por la rama incisiva de la arteria alveolar inferior (Flanagan). Con esto en cuenta, surge la siguiente pregunta: Si se produce algún cambio en la formula dentaria del sector mandibular anterior, ¿Se verá alterada la morfología y longitud de los forámenes y canales óseo linguales relacionados con las espinas mentales?

\footnotetext{
* Unidad de Morfología, Facultad de Odontología, Universidad de los Andes, Santiago, Chile.

** División de Ciencia, Investigación y Gestión, Facultad de Odontología, Universidad de Los Andes, Chile.
} 
En la literatura existente, no se alusión a la relación entre la presencia de estos forámenes y el grado de desdentamiento. Lautner et al. (2001), evaluó la relación entre el grado de atrofia del proceso alveolar postexodoncia y la posición del foramen lingual, encontrando que a mayor desdentamiento, el foramen lingual se acercaba cada vez más al vértice del proceso alveolar e incluso en mandíbulas extremadamente reabsorbidas, este foramen se encontró por vestibular. De todas formas no realizó una diferencia entre los distintos forámenes ubicados en la línea mediana ni tampoco evaluó el diámetro y longitud de estos en relación al grado de desdentamiento.

El objetivo de este estudio fue evaluar la relación entre la permanencia de dientes y la presencia y morfometría de forámenes y canales ubicados en cercanía a las espinas mentales.

\section{MATERIAL Y MÉTODO}

Se llevó a cabo un estudio de tipo transversal seleccionando un total 95 mandíbulas humanas secas, con sexo y edad indeterminada, de la Unidad de Morfología de la Universidad de los Andes y del Departamento de Anatomía y Biología del Desarrollo del Instituto de Ciencias Biomédicas de la Universidad de Chile. Estás fueron enumeradas con un lápiz de tinta permanente en la región de la fosita sublingual izquierda de forma aleatoria desde el número 1 hasta el 95. Se desarrolló una base de datos con el programa Excel ${ }^{\circledR}$ donde se anotaron correlativamente los siguientes datos: número de dientes remanentes, número de dientes remanentes en el grupo $\mathrm{V}$, número de forámenes en la línea mediana, número de forámenes superiores a las espinas mentales superiores (FSEM), número de forámenes entre las espinas mentales superiores (FEEMS), número de forámenes entre las espinas mentales superiores e inferiores (FEEM), número de forámenes entre las espinas mentales inferiores (FEEMI), número de forámenes inferiores a las espinas mentales inferiores (FIEM), áreas correspondiente a cada uno de los forámenes descritos anteriormente y finalmente las longitudes de los canales que se desprenden de los forámenes. La recolección de los datos se realizó mediante una inspección visual a través de un operador. Para obtener las áreas y longitudes se procedió a fijar un pedazo de papel milimetrado de $2 \mathrm{~cm}$. de alto por $1 \mathrm{~cm}$. de ancho inmediatamente hacia la derecha de la línea mediana para cada mandíbula (Figs. 1 y 2). Una vez completada esta etapa se obtuvo fotos estandarizadas utilizando una cámara Cibershot DscS75 y un trípode. Se obtuvo 2 imágenes por cada mandíbula, una imagen para los forámenes ubicados superiormente y otra para los forámenes ubicados inferiormente. Se tuvo

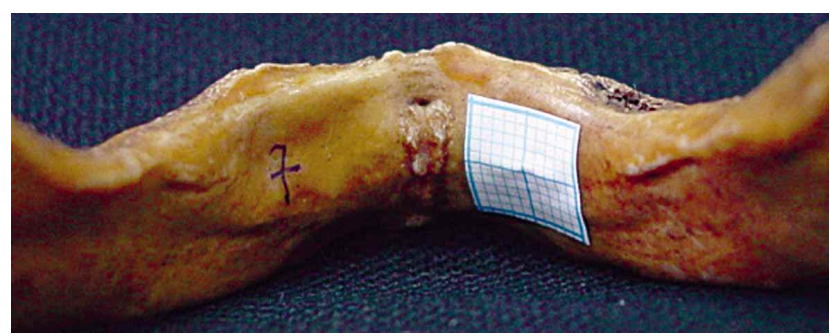

Fig. 1. Mandíbula dentada en el grupo V con numeración y papel milimetrado.

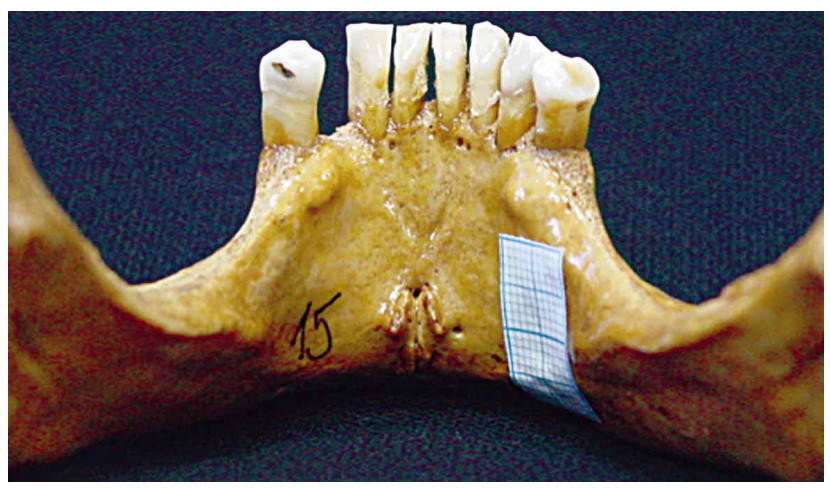

Fig. 2. Mandíbula desdentada en el grupo V con numeración y papel milimetrado.

la precaución de lograr una imagen ortográfica de los forámenes en estudio. Luego de esto se procedió a introducir una lima $\mathrm{k}$ de subserie correspondiente a la lima 0,8 . Se introdujo en cada foramen hasta constatar tope óseo y se llevó a posición el tope de goma. Esta lima luego se colocó sobre un papel milimetrado en el que se encontraba escrito el número de la mandíbula y a qué foramen correspondía. Una vez ubicada de esta manera, se obtuvo una fotografía con la misma cámara utilizada anteriormente. Finalmente estas imágenes fueron analizadas utilizando el programa Photoshop ${ }^{\circledR}$ Cs 3 Extended. Como este programa mide en pixeles, basándonos en el papel milimetrado que se fotografió pegado en la mandíbula, se logró trasformar los pixeles a milímetros. Para la medición del área correspondiente a cada uno de los forámenes, se procedió a seleccionar manualmente la zona en el programa y se utilizó la herramienta "Record Measurements" donde nos entrega el tamaño en mm2 del área seleccionada. Para el caso de las imágenes de las limas también se realizó la conversión de milímetros a pixeles y utilizando la herramienta "Ruler Tool" se constató la longitud de cada canal, tabulando esta información en la base de datos. Con esto se obtuvo la descripción de la muestra (cantidad de dientes remanentes, cantidad de dientes remanentes en grupo $\mathrm{V}$, número de forámenes, distribución de estos respecto de las espinas mentales, áreas y longitudes de los forámenes). Además se relacionó la presencia y ausencia de dientes en el grupo 5 con el diámetro y longitud de cada una de las posibles posiciones de los canales y 
forámenes. Se consideró una mandíbula dentada en el grupo $\mathrm{V}$ a la que presentara 4 o más dientes en esta zona y se consideró desdentada aquella que tuviese la ausencia total de dientes en el grupo V. Se utilizó test de Shapiro Wilk para evaluar la normalidad de la muestra. Cuando la distribución de los datos fue normal se utilizó la prueba t para muestras independientes y cuando la distribución no fue normal se utilizó test de Mann Whitney.

\section{RESULTADOS}

La descripción de las variables: número de dientes remanentes, número de dientes remanentes en el grupo V, número de forámenes, distribución según posición de los forámenes, se encuentra detallada en la Tabla I. Se obtuvo un total de 53 mandíbulas dentadas en el grupo V y 42 mandíbulas desdentadas en el grupo V. La descripción de la muestra respecto al área de cada foramen según su posición en la línea mediana se describe en la Tabla II. Para el caso de los forámenes inferiores a las espinas mentales inferiores (FIEM) se encontraron mandíbulas que presentaron dos (29 especímenes) e incluso tres forámenes en esta posición (2 especímenes). Por esta razón los forámenes inferiores a las espinas mentales inferiores fueron enumerados desde el 1 al 3 según su orden desde superior a inferior para la descripción. Idéntica forma de describir la muestra se utilizó para las longitudes de los canales óseos asociados a estos forámenes (Tabla III).

Cuatro mandíbulas no presentaron forámenes en la línea mediana lingual. El 95,7\% de la muestra presentó por lo menos 1 foramen en relación a las espinas mentales con un máximo de 5 forámenes en esta zona (Tabla I). Se observa que los forámenes con mayor aparición fueron los FSEM en 75 mandíbulas, FIEM en 72 mandíbulas (incluso encontrando 2 forámenes inferiores en 28 casos y tres forámenes inferiores en 2 casos) y FEEM con 26 mandíbulas. Debido a que la frecuencia de distribución de los forámenes se encuentra principalmente en estas ubicaciones, se consideraron estas tres posiciones para comparar el comportamiento del área y longitud de los forámenes y canales linguales entre mandíbulas dentadas y desdentadas en el grupo V.

En la Tabla IV se entrega la descripción de las áreas de los FSEM, FEEM y de los FIEM para las mandíbulas dentadas en el grupo $\mathrm{V}$ y desdentadas en el grupo $\mathrm{V}$ respectivamente. Para comparar las áreas entre dentados en el grupo $\mathrm{V}$ y desdentado en el grupo V se utilizó test de Mann Whitney debido a la ausencia de distribución normal en la muestra. En caso del FSEM se obtuvo un valor p de 0,42, para el FEEM se obtuvo un valor $\mathrm{p}$ de 0,187 y para el FIEM se obtuvo un valor p de 0,0194 (siendo de mayor área los forámenes de mandíbulas dentadas en el grupo V).

En la Tabla V se entrega la descripción de las longitudes de los canales SEM, EEM, IEM para las mandíbulas dentadas en el grupo $\mathrm{V}$ y desdentadas en el grupo $\mathrm{V}$ respectivamente. En esta ocasión, para comparar las longitudes entre dentados en el grupo V y desdentado en el grupo V se utilizó test de Mann Whitney debido a la ausencia de distribución normal en el caso de canales SEM e IEM. En el caso del canal EEM se utilizó prueba t para muestras independientes. Para el canal SEM se obtuvo un valor p de 0,0206 (siendo de mayor longitud los canales de mandíbulas desdentadas), para el canal EEM se obtuvo un valor p de 0,079 y para el canal IEM se obtuvo un valor $\mathrm{p}$ de 0,722 .

Tabla I. Descripción del número de dientes remanentes, dientes remanentes en el grupo V, número de forámenes en la línea mediada y los distintos forámenes descritos en las 95 mandíbulas estudiadas.

\begin{tabular}{lcccc}
\hline Variable & Mediana & $\begin{array}{c}\text { Recorrido } \\
\text { Intercuartílico }\end{array}$ & Mín. & Máx. \\
\hline Número de dien tes remanentes & 6 & 10 & 0 & 16 \\
Dientes remanente en grupo V & 4 & 6 & 0 & 6 \\
Número de agujeros & 2 & 1 & 0 & 5 \\
FSEM & 1 & 0 & 0 & 1 \\
FEEMS & 0 & 0 & 0 & 1 \\
FEEM & 0 & 1 & 0 & 1 \\
FEEMI & 0 & 0 & 0 & 1 \\
FIEM & 1 & 1 & 0 & 3 \\
\hline
\end{tabular}

(FSEM: foramen superior a espinas mentales, FEEMS: foramen entre espinas mentales superiores, FEEM: foramen entre espinas mentales, FEEMI: foramen entres espinas mentales inferiores, FIEM: foramen inferior a espinas mentales). 
CÁCERES, F.; RAMíREZ V. \& SOTO, R. Influencia de dientes remanentes en la presencia y morfometría de forámenes y canales en relación a las espinas mentales Int. J. Morphol., 32(1):106-111, 2014.

Tabla II. Descripción de las áreas obtenidas para cada uno de los forámenes.

\begin{tabular}{lccccccc}
\hline Área & Promedio & $\begin{array}{c}\text { Desviación } \\
\text { estándar }\end{array}$ & Mediana & $\begin{array}{c}\text { Recorrido } \\
\text { Intercuartílico }\end{array}$ & Mín. & Máx. & $\mathbf{n}$ \\
\hline FSEM & 0,441 & 0,308 & 0,361 & 0,328 & 0,092 & 2,02 & 75 \\
FEEMS & 0,417 & 0,122 & 0,378 & 0,064 & 0,319 & 0,628 & 5 \\
FEEM & 0,367 & 0,315 & 0,284 & 0,328 & 0,034 & 1,527 & 26 \\
FEEMI & 0,357 & 0,366 & 0,291 & 0,314 & 0,037 & 1,254 & 9 \\
FIEM1 & 0,484 & 0,421 & 0,351 & 0,354 & 0,063 & 2,1 & 72 \\
FIEM2 & 0,381 & 0,261 & 0,256 & 0,364 & 0,024 & 0,952 & 28 \\
FIEM3 & 0,405 & 0,255 & 0,405 & 0,361 & 0,224 & 0,585 & 2 \\
\hline
\end{tabular}

(FSEM: foramen superior a espinas mentales, FEEMS: foramen entre espinas mentales superiores, FEEM: foramen entre espinas mentales, FEEMI: foramen entres espinas mentales inferiores, FIEM 1, 2,3: foramen inferior a espinas mentales).

Tabla III. Descripción de las longitudes obtenidas para cada uno de los canales estudiados.

\begin{tabular}{lccccccc}
\hline Longitud & Promedio & $\begin{array}{c}\text { Desviación } \\
\text { estándar }\end{array}$ & Mediana & $\begin{array}{c}\text { Recorrido } \\
\text { Intercuartílico }\end{array}$ & Mín. & Máx. & n \\
\hline SEM & 6,874 & 3,271 & 6,44 & 4,18 & 1,32 & 15 & 75 \\
EEMS & 7,744 & 3,834 & 6,9 & 3,87 & 2,5 & 12,45 & 5 \\
EEM & 6,109 & 3,084 & 6,25 & 4,18 & 0,7 & 11,23 & 26 \\
EEMI & 5,938 & 3,138 & 5,7 & 6,3 & 1,7 & 9,6 & 9 \\
IEM1 & 5,795 & 2,789 & 5,375 & 5,1 & 1,5 & 12,57 & 72 \\
IEM2 & 4,586 & 2,357 & 4,41 & 3 & 0 & 11,7 & 28 \\
IEM3 & 5,39 & 1,138 & 5,5 & 2,27 & 4,2 & 6,47 & 2 \\
\hline
\end{tabular}

(SEM: canal superior a espinas mentales, EEMS: canal entre espinas mentales superiores, EEM: canal entre espinas mentales, EEMI: canal entres espinas mentales inferiores, IEM 1, 2,3: canal inferior a espinas mentales).

Tabla IV. Descripción del área de los forámenes para mandíbulas desdentadas y dentadas en el grupo V.

\begin{tabular}{lcccccc}
\hline Variables & $\begin{array}{c}\text { Área FSEM } \\
\text { Desdentado } \\
\text { Grupo V (26) }\end{array}$ & $\begin{array}{c}\text { Área FSEM } \\
\text { Dentado } \\
\text { grupo V (43) }\end{array}$ & $\begin{array}{c}\text { Área FEEM } \\
\text { Desdentado } \\
\text { Grupo V (10) }\end{array}$ & $\begin{array}{c}\text { Área FEEM } \\
\text { Dentado } \\
\text { Grupo V (12) }\end{array}$ & $\begin{array}{c}\text { Área FIEM } \\
\text { Desdentado } \\
\text { Grupo V (41) }\end{array}$ & $\begin{array}{c}\text { Área FIEM } \\
\text { Dentado } \\
\text { Grupo V (54) }\end{array}$ \\
\hline Prome dio & 0,355 & 0,451 & 0,227 & 0,469 & 0,417 & 0,497 \\
DE & 0,183 & 0,345 & 0,104 & 0,417 & 0,423 & 0,368 \\
Mediana & 0,356 & 0,358 & 0,24 & 0,406 & 0,24 & 0,383 \\
RIC & 0,277 & 0,357 & 0,157 & 0,439 & 0,406 & 0,331 \\
\hline
\end{tabular}

(FSEM: foramen superior a espinas mentales, FEEM: foramen entre espinas mentales, FIEM: foramen inferior a espinas mentales, DE: desviación estándar, RIC: recorrido intercuartílico).

Tabla V. Descripción de la longitud de los canales para mandíbulas desdentadas y dentadas en el grupo V.

\begin{tabular}{lcccccc}
\hline Variables & $\begin{array}{c}\text { Longitud SEM } \\
\text { Desdentado } \\
\text { Grupo V (26) }\end{array}$ & $\begin{array}{c}\text { Longitud SEM } \\
\text { Dentado grupo } \\
\text { V (43) }\end{array}$ & $\begin{array}{c}\text { Longitud EEM } \\
\text { Desdentado } \\
\text { Grupo V (10) }\end{array}$ & $\begin{array}{c}\text { Longitud EEM } \\
\text { Dentado } \\
\text { Grupo V (12) }\end{array}$ & $\begin{array}{c}\text { Longitud IEM } \\
\text { Desdentado } \\
\text { Grupo V (42) }\end{array}$ & $\begin{array}{c}\text { Longitud IEM } \\
\text { Dentado } \\
\text { Grupo V (51) }\end{array}$ \\
\hline Prome dio & 8,185 & 6,145 & 7,38 & 5,031 & 5,525 & 5,643 \\
DE & 3,378 & 2,99 & 5,598 & 3,244 & 2,374 & 3,034 \\
Mediana & 7,38 & 5,3 & 7,265 & 4,575 & 4,98 & 5 \\
RIC & 4,77 & 5,01 & 3,21 & 5,525 & 3,32 & 5,3 \\
\hline
\end{tabular}

(SEM: canal superior a espinas mentales, EEM: canal entre espinas mentales, IEM: canal inferior a espinas mentales, DE: desviación estándar, RIC: recorrido intercuartílico). 
Finalmente, utilizando un modelo de regresión logística, se evaluó la influencia del desdentamiento o presencia de dientes en el grupo $\mathrm{V}$ en la aparición del foramen para cada posición. No se observó asociación significativa entre la presencia o ausencia de dientes con la aparición de forámenes para las tres posiciones evaluadas; FSEM con un OR de 0,595 y un valor p de 0,307, FEEM con un OR de 1,14 y un valor $p$ de 0,778 y para los FIEM con un OR de $1,049 \mathrm{y}$ un valor $\mathrm{p}$ de 0,922 .

\section{DISCUSIÓN}

La presencia de forámenes y canales en la región anterior mandibular se ha descrito en una gran cantidad de publicaciones, demostrando que la presencia de estos forámenes varía según los distintos autores entre un 32\% hasta incluso un $100 \%$ de las muestras analizadas (McDonnell et al.; Gahleitner et al., 2001; Nagar et al., 2001; Przystanska \& Bruska, 2005; Liang et al., 2006; Soto et al.). Además se ha descrito que el contenido que se encuentra en este reparo anatómico consiste en tejido nervioso y principalmente vasos sanguíneos (McDonnell et al.; Liang et al., 2005), siendo el sentido del flujo sanguíneo fundamentalmente desde lingual hacia la profundidad del tejido óseo (Lustig et al.). Finalmente la importancia de estos forámenes linguales radica en el peligro relacionado con la ruptura de estos durante procedimientos quirúrgicos, produciendo hematomas sublinguales que pueden llevar incluso al compromiso de la vía aérea (Flanagan; Mraiwa et al., 2003; Kalpidis \& Setayesh; Tagaya et al., 2009).

Uno de los objetivos de este estudio fue continuar el estudio realizado con anterioridad por este equipo mostrando los resultados obtenidos con una mayor muestra (Soto et al.). El 95,78 \% de nuestra muestra presento uno o más forámenes a nivel lingual de la cortical mandibular, siendo los más comunes los FSEM encontrados en un 78,4\% de la muestra, FEEM encontrados en un 27,36\% de la muestra y FIEM encontrados en $75,78 \%$ de la muestra, confirmando la tendencia observada por el estudio anterior y concordando con la mayor presencia de forámenes sobre y bajo las espinas mentales descritos por otros autores (McDonnell et al.; Liang et al.). Nagar et al. en una muestra de población india, también menciona la alta prevalencia del FSEM con una presencia en un $72,45 \%$ de su muestra, sin embargo, la cantidad de FIEM encontrados por su estudio solo llega al 5,98. Una situación interesante encontrada por nuestro estudio fue que el desdentamiento o la presencia de dientes en el grupo $\mathrm{V}$ no afectó la frecuencia de forámenes para ninguna de las posiciones estudiadas, situación similar a la descrita por Vasconcellos et al. (2000), donde los porcentajes de forámenes linguales no variaron mayormente entre mandíbulas dentadas y desdentadas.
Respecto a la relación entre el tamaño de los forámenes y la longitud de los canales con la presencia y ausencia de dientes; no existen en la literatura artículos similares a los realizados por este equipo. Los resultados obtenidos muestran que el área de los forámenes superiores a las espinas mentales y entre las espinas mentales no guarda mayor relación con la presencia o no de dientes en el grupo V. Situación diferente al área de los forámenes inferiores, donde el grupo dentado tendía a tener mayor área de forámenes que el grupo desdentado con un valor $p$ de 0,0194 . Si tomamos ahora en cuenta la longitud de los canales estudiados, se encontró que los canales superiores a las espinas mentales superiores fueron de mayor longitud en las mandíbulas desdentadas que en las mandíbulas dentadas con un valor p de 0,0206. Para las demás posiciones analizadas (áreas y longitudes de forámenes y canales ubicados entre las espinas mentales e inferiores a las espinas mentales inferiores) no existió diferencia estadísticamente significativa entre mandíbulas dentadas y desdentadas en el grupo V.

Por la posición de los ápices dentarios podemos pensar que los vasos que entran por la tabla lingual mandibular en la línea mediana podrían estar destinados principalmente al tejido óseo, al no producirse una degeneración del canal óseo postexodoncia de estos dientes. La mayor área encontrada en los forámenes inferiores en mandíbulas dentadas comparadas con las desdentadas se puede deber a una labor nutricia complementaria a la irrigación proveniente de la arteria alveolar inferior para los dientes anteroinferiores. Ahora, si tomamos en cuenta la longitud de los canales, observamos que el canal superior a las espinas mentales superiores presentó estadísticamente una mayor longitud en mandíbulas desdentadas en el grupo V. Si consideramos los vasos que se encuentran entrando por superior a las espinas mentales como aporte principalmente óseo, es lógico pensar que con la degeneración de los vasos alveolares inferiores en esta zona por el desdentamiento reciban un aporte complementario por parte de vasos linguales, destinado a zonas más vestibulares del hueso remanente en el grupo V. Lamentablemente con los resultados de este estudio no podemos afirmar de manera categórica estas ideas, pero es importante tener en cuenta que el desdentamiento no afectó la frecuencia de la aparición de estos forámenes en ninguna de las posiciones estudiadas. Con esto podemos concluir que el estudio de estos forámenes y canales previo a cirugías en relación a la línea mediana mandibular debe ser preciso para cada caso. Desde un implante unitario en la zona en pacientes desdentados parciales hasta tratamiento en base a múltiples implantes, profundizaciones de flanco lingual, etc. presentan el mismo riesgo de accidentes vasculares, por lo que se debe tener en cuenta la presencia de estos reparos anatómicos y los vasos sanguíneos que por ellos transcurren para evitar accidentes graves. 
CÁCERES, F.; RAMÍREZ V. \& SOTO, R. Influence of remnant teeth in the presence and morphometry of foramina and canals in relation to mental spine. Int. J. Morphol., 32(1):106-111, 2014.

SUMMARY: The anterior mandibular body is an anatomical area that is often subjected to surgery such as for installation of osseointegrated implants, mentoplasty, genioplasty, fractures, among others. The presence of vessels that penetrates through foraminas at its midline from the lingual table has been described. Tooth loss in the anterior mandibular zone may influence the size of the foramina and canals due to the atrophy of incisors vessels that originates from the inferior alveolar artery. The aim was to evaluate the relationship between presence and absence of remaining teeth in group V, with the presence and morphometry of foramina and canals located in proximity to the mental spines. We studied 95 dry human mandibles with indeterminate sex and age. We measured the area of the foraminas and the length of the canals using the program Photoshop CS3 Extended. We obtained a total of 53 dentate and 42 edentulous mandibles in group V. In $95.7 \%$ of the sample had the presence of at least one foramina relative to the mental spines. The foramina that was found more often, tended to be upper to the superior mental spines, in a total of 75 mandibles; lower to the inferior mental spines in 72 mandibles and between the mental spines in 26 mandibles. There was no association between the presence and absence of teeth versus the frequency of the foramina in the three positions established. It was seen that the foramina lower to the inferior mental spine had a greater diameter in dentate mandibles $(\mathrm{p}$-value $=$ 0.0194 ) and the canal upper to the superior mental spines was significantly longer in the edentulous mandibles ( $\mathrm{p}$-value $=0.079$ ).

KEY WORDS: Lingual foramen; Lingual canal; Mental spine.

\section{REFERENCIAS BIBLIOGRÁFICAS}

Flanagan, D. Important Arterial Supply of the Mandible, Control of an Arterial Hemorrhage, and Report of a Hemorrhagic Incident. J. Oral Implantol., 29(4):165-73, 2003.

Gahleitner, A.; Hofschneider, U.; Tepper, G.; Pretterklieber, M.; Schick, S.; Zauza, K. \& Watzek, G. Lingual Vascular Canals of the Mandible: Evaluation with Dental CT. Radiology, 220(1):186-9, 2001.

Isaacson, T. J. Sublingual Hematoma Formation During Immediate Placement of Mandibular Endosseous Implants. J. Am. Dent. Assoc., 135(2):168-72, 2004.

Kalpidis, C. D. \& Setayesh, R. M. Hemorrhaging Associated with Endosseous Implant Placement in the Anterior Mandible: A Review of the Literature. J. Periodontol., 75(5):631-45, 2004.

Lautner, N. V.; Krenkel, C.; Gaggl, A. \& Bernauer, E. Altered Position of the Medial Lingual Nutritional Foramina in the Different Stages of Alveolar Ridge Atrophy. J. Oral Implantol., 2011.

Liang, H.; Frederiksen, N. L. \& Benson, B. W. Lingual Vascular Canals of the Interforaminal Region of the Mandible: Evaluation with Conventional Tomography. Dentomaxillofac. Radiol., 33(5):340-1, 2004.

Liang, X.; Jacobs, R. \& Lambrichts, I. An Assessment on Spiral Ct Scan of the Superior and Inferior Genial Spinal Foramina and Canals. Surg. Radiol. Anat., 28(1):98-104, 2006.

Liang, X.; Jacobs, R.; Lambrichts, I.; Vandewalle, G.; van Oostveldt, D.; Schepers, E.; Adriaensens, P. \& Gelan, J. Microanatomical and Histological Assessment of the Content of Superior Genial Spinal Foramen and Its Bony Canal. Dentomaxillofac. Radiol., 34(6):3628,2005 .

Lustig, J. P.; London, D.; Dor, B. L. \& Yanko, R. Ultrasound Identification and Quantitative Measurement of Blood Supply to the Anterior Part of the Mandible. Oral Surg. Oral Med. Oral Pathol. Oral Radiol. Endod., 96(5):625-9, 2003.

McDonnell, D.; Reza Nouri, M. \& Todd, M. E. The Mandibular Lingual Foramen: A Consistent Arterial Foramen in the Middle of the Mandible. J. Anat., 184(Pt. 2):363-9, 1994.
Mraiwa, N.; Jacobs, R.; van Steenberghe, D. \& Quirynen, M. Clinical Assessment and Surgical Implications of Anatomic Challenges in the Anterior Mandible. Clin. Implant Dent. Relat. Res., 5(4):219-25, 2003.

Nagar, M.; Bhardwaj, R. \& Prakash, R. Accessory Lingual Foramen in Adult Indian Mandibles. J. Anat. Soc. India, 50(1):13-14, 2001.

Przystanska, A. \& Bruska, M. Foramina on the Internal Aspect of the Alveolar Part of the Mandible. Folia Morphol. (Warsz), 64(2):8991, 2005.

Romanos, G. E.; Gupta, B. \& Crespi, R. Endosseous arteries in the anterior mandible: literature review. Int. J. Oral Maxillofac. Implants, 27(1):90-4, 2012

Rosano, G.; Taschieri, S.; Gaudy, J. F. Testori, T. \& Del Fabbro, M. Anatomic assessment of the anterior mandible and relative hemorrhage risk in implant dentistry: a cadaveric study. Clin. Oral Implants Res., 20(8):791-5, 2009.

Soto, R.; Cáceres, F. \& García, R. Presence and Morphometry of Foramina and Canals in Relation to Mental Spines. Int. J. Morphol., 30(2):41721, 2012.

Tagaya, A.; Matsuda, Y.; Nakajima, K.; Seki, K. \& Okano, T. Assessment of the blood supply to the lingual surface of the mandible for reduction of bleeding during implant surgery. Clin. Oral Implants Res., 20(4):351-5, 2009.

Vasconcellos, H. A.; Siqueira C. A. E.; Almeida, G. H.; Maia, M. L. T. \& Barros, de V. P. H. Anatomía del canal y foramen lingual y sus relaciones con la sínfisis mandibular. Rev. Chil. Anal, 18(1):47-51, 2000.

Dirección para Correspondencia:

Reinaldo Soto Norambuena

Universidad de los Andes

Monseñor Álvaro del Portillo 12455

Las Condes, Santiago

CHILE

Email: rsoto@miuandes.cl

Recibido : 04-06-2013

Aceptado: 19-11-2013 